\title{
Water clusters in plants. Fast channel plant communications. Planet influence
}

\author{
Kristina Zubow ${ }^{1}$, Anatolij Viktorovich Zubow ${ }^{2}$, Viktor Anatolijevich Zubow ${ }^{1 *}$ \\ 1““Aist Handels- und consulting GmbH”, R\&D Department, Groß Gievitz, Germany; *Corresponding Author: aist@zubow.de \\ ${ }^{2}$ Department of Computer Science, Humboldt University Berlin, Berlin, Germany; zubow@informatik.hu-berlin.de
}

Received 19 April, 2010; revised 30 April, 2010; accepted 7 May 2010.

\section{ABSTRACT}

In tubers of two potato cultivars and in one apple cultivar, water clusters, consisting of $11 \pm 1$, $100,178,280,402,545,715,903,119,1351,1606$ and 1889 molecules, were directly (in-vivo) analyzed by gravitation spectroscopy. The clusters' interactions with their surroundings during plant growth in summer 2006 in Germany were described where a model represents the states of water clusters in bio matrices. Furthermore, a comparison with clusters in irrigation water (river, rain) is given. To achieve a high and good quality yield it is necessary to choose the right irrigation water that has to correspond with the water cluster super structure in plants. The formation energy for the $\left(\mathrm{H}_{2} \mathrm{O}\right)_{280}$ cluster during plant growth is between 0.4 and $1.3 \mathrm{~kJ} / \mathrm{mol}$. Water clusters were found to communicate with surroundings by resonance field oscillations. The main cluster parameters which were investigated are intensities of oscillations, average molecular masses, rate of collapsed clusters, and total number of clusters in ensemble during potato (apple) growth. A correlation between the change of water cluster ensembles in plants with molecular masses of all clusters in isolated starch (in-vitro) during plant growth process is discussed. In particular for potato tubers' growth, there was observed a correlation between water cluster development and average molecular masses of amylopectin super coils. The communication of plants with each other and with surroundings proceeds by resonance field of oscillating water clusters. Planet gravitation was found to influence the water cluster structure in plants.

Keywords: Clusters; Field; Communication; Gravitation; Spectroscopy; Planets

\section{INTRODUCTION}

It is well known, that in the bulk water molecules form clusters [1]. Though clusters were discovered in biological matrices [2] until now there isn't still a method by which clusters in plants can be identified during growth in-vivo. By Okonchi Shoichi [3] it was suggested, that water molecules are in the form of clusters in living organisms. In Figure 1 computer models of some water clusters are given. In our laboratory, we developed a gravitation spectrometer for water cluster identification in bio-matrices of plants [4-6]. Knowing the state of water clusters in plants could be helpful for understanding the relation between biochemical processes at nanoscale level during growth and qualitative yields.

The aim of the present work was to investigate water cluster ensembles in potato tubers and apples during their growth period in summer 2006 furthermore, to understand whether a communication of clusters with each other or with their surroundings is possible.

\section{MATERIAL AND METHODS}

Potato starch from different cultivars ("Agria-N" from Kartoffelzucht Böhm KG and "Kalena" from NORIKA Nordring-Kartoffelzucht-und Vermehrungs-GmbH, both of Germany) prepared by a standard method was chosen as investigation object. Pure amylopectin was provided by Bavaria State Research Center LfL. The mass spectra were measured with the gravitation spectrometer (GS) of the Aist Handels- und Consulting $\mathrm{GmbH}$, Germany (www.zubow.de), where the energy ( $f$ ) caused by the interaction of the clusters with a shock wave is a function of molecular masses of the oscillating clusters. Molecular masses of clusters were calculated by Zubow Equations [5]. The calibration of the device was carried out with the clusters $\left(\mathrm{H}_{2} \mathrm{O}\right)_{11-12}$ [7-9], $\left(\mathrm{H}_{2} \mathrm{O}\right)_{100}$ and $\left(\mathrm{H}_{2} \mathrm{O}\right)_{280}$ both by Chaplin [10] in bi-distilled water at 295 $\mathrm{K}$ [1]. The potato samples (tubers) were taken at the very same place (planted on 27.04.2006) whereas the apple 


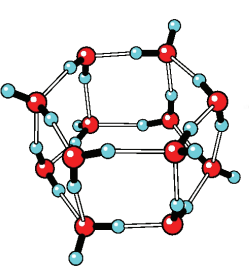

$\left(\mathrm{H}_{2} \mathrm{O}\right)_{12}$

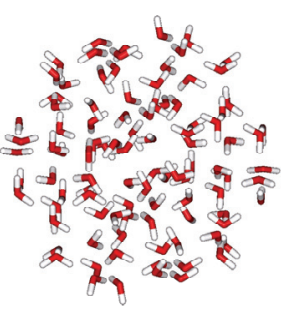

$\left(\mathrm{H}_{2} \mathrm{O}\right)_{100}$

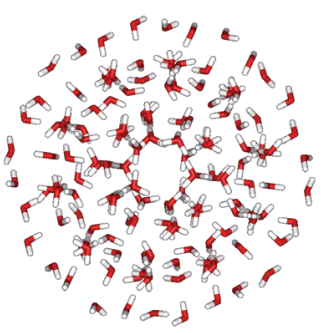

$\left(\mathrm{H}_{2} \mathrm{O}\right)_{160}$

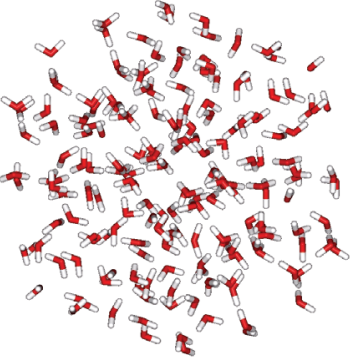

$\left(\mathrm{H}_{2} \mathrm{O}\right)_{184}$

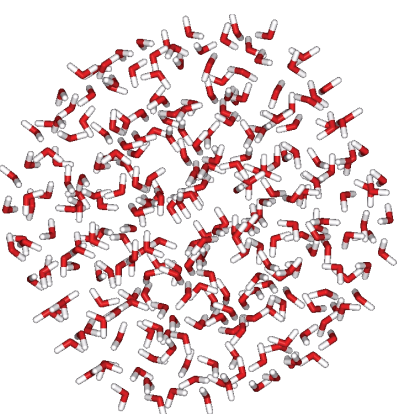

$\left(\mathrm{H}_{2} \mathrm{O}\right)_{280}$

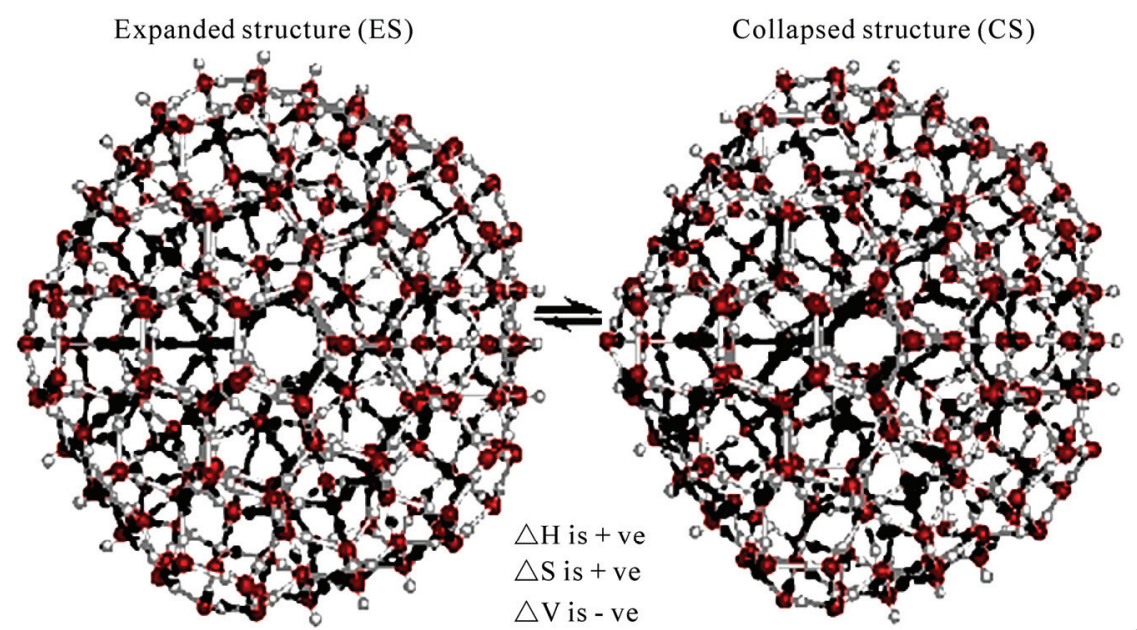

Figure 1. Calculated water cluster models (with kind permission of Prof. Chaplin [10] and Prof. Lenz A. [9] hydrogen bonds were shown for $\left(\mathrm{H}_{2} \mathrm{O}\right)_{12}$ only.

sample of "alstar" cultivar was withdrawn at the very same tree and at the very same branch. Pure agarose of Aldrich $\left(\mathrm{T}_{\mathrm{gel}}=315 \mathrm{~K}, \mathrm{~T}_{\mathrm{fl}}=533 \mathrm{~K}\right)$ was used for preparing a model bio-matrix. Agarose hydrogels were prepared by dissolving agarose $(0.3,0.8$ and $3 \mathrm{wt}$. \%) in boiling distilled water $\left.(15 \pm 5) \cdot 10^{-8}, \mathrm{om}^{-1} * \mathrm{~cm}^{-1}\right)$ with following cooling until room temperature. Below the general Scheme 1 of the GS-measuring cell for the investigation of clusters in samples is shown [6]. The average day temperature $\left(\mathrm{T}_{\mathrm{d}}\right.$, from 09.00 a.m. until 06.00 p.m.) between June $8^{\text {th }}\left(\mathrm{x}_{0}=1987\right)$ and September $1^{\text {st }}\left(\mathrm{x}_{i}=2070\right)$ can be described with $\mathrm{T}_{\mathrm{d}}=3 \mathrm{E}-05 \cdot \mathrm{x}^{3}-0.1889 \cdot \mathrm{x}^{2}+$ 393.73 $x$ - 273034.

The GMS-method called in earlier works of authors as FNS-method is described in detail in [1,5], (algorithmus

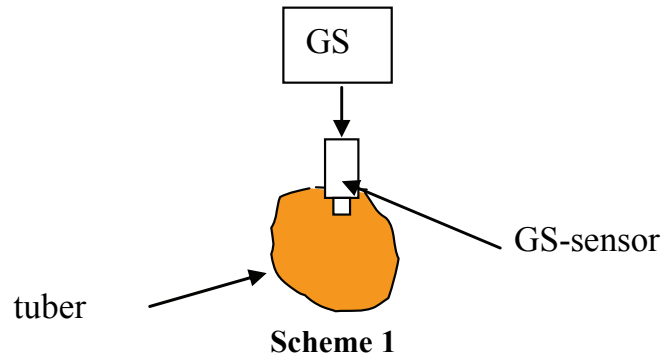

see in Figure 2). The negative value of a signal in the

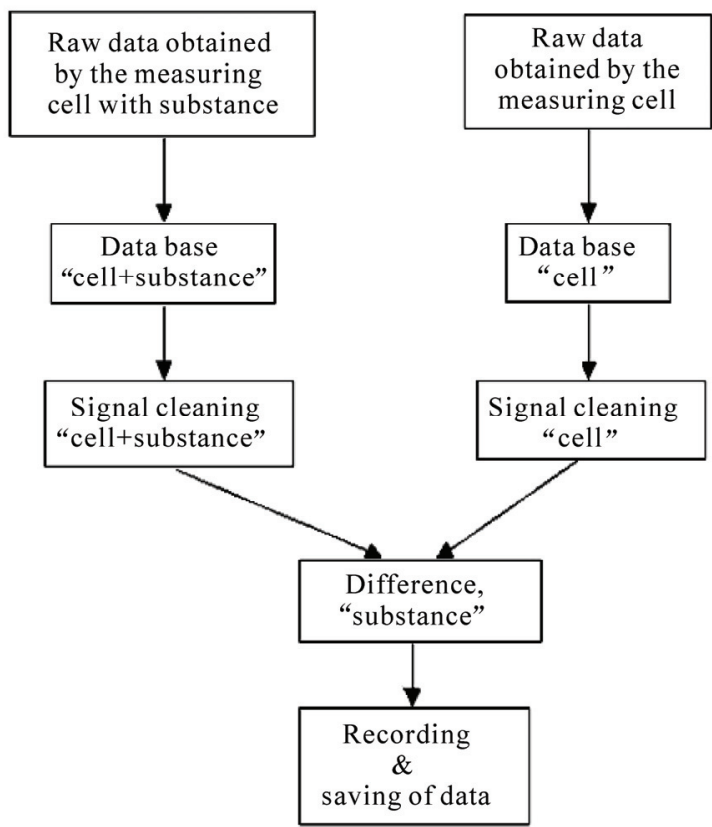

Figure 2. Secondary data extraction of GMS signals. 
GS spectrum means energy absorption, which is necessary for melting of the collapsed cluster. On the other side, the positive signal is an energy release at the interaction of the shock wave with the expanded cluster, leading to its destruction. The signals of collapsed clusters were therefore described with minus and those of expanded one with plus. The Zayzev program Astronet, version ZET 9 (www.astrozet.net) was used to get information on the planet positions.

\section{RESULTS AND DISCUSSION}

Typical GS-spectra for rain and river water and water in potato tuber tissue are shown in Figure 3. As visible all clusters modeled in Figure $\mathbf{1}$ are present here. Water clusters in materials were found to be in different forms (collapsed or expanded), which depends on the interaction of clusters with their surroundings [1]. In this study, we report the analysis data of water clusters $(12,100$, 178,280 and 1889) in potato tubers and apples during their growth in North Germany in 2006. The signal of clusters with $11 \pm 1$ water molecules is stable in all investigated samples and the expanded form $(+f)$ of clusters is dominating over the collapsed one $(-f)$. Remarkably, that the collapsed form is more enriched with energy than the expanded one.

The cluster of 100 water molecules in examined potatoes is in its collapsed form however in river water this cluster is expanded and in rainwater it is often missing. This means that the plants need energy to adapt the irrigation water cluster structure (river, lake, rain etc.) to

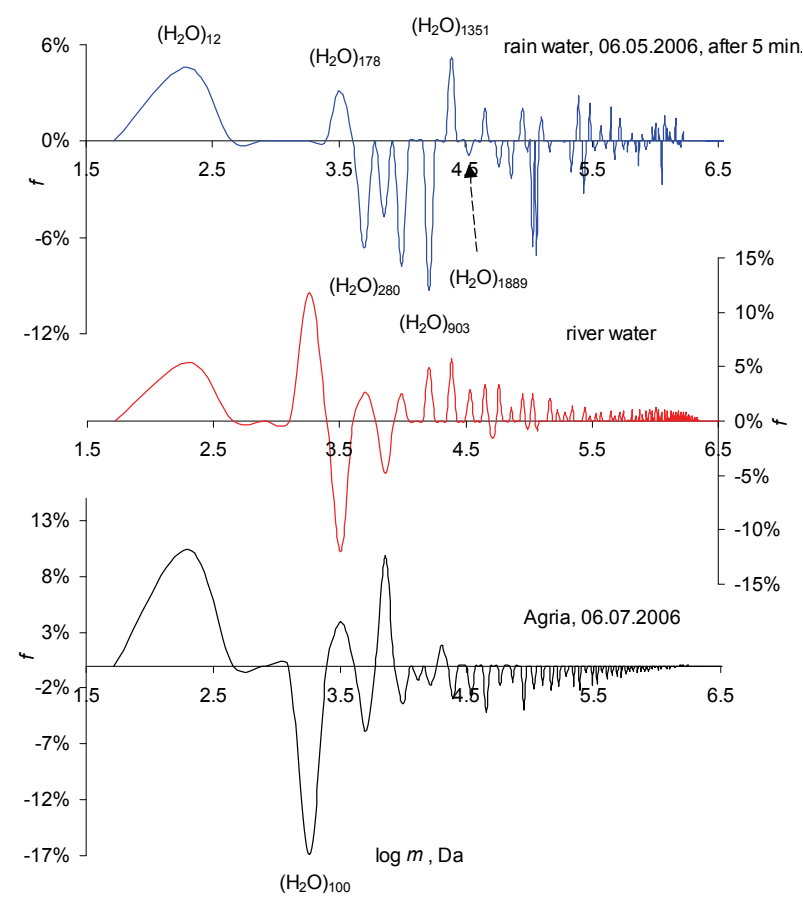

Figure 3. GM-spectra of water in different substances. the water clusters in bio matrices. Irrigation water must have similar water cluster (long-range order) forms like those occurring in plants logically. It is therefore assumed that the irrigation water weakens the immune system of the plants and reduces indirectly their energy potential.

In Figure 4, the development of all clusters (water, bio molecules, solvated clusters of ion pairs [1,11] and bio polymers $[2,12]$ in plants during growth in summer 2006 is shown. There are strong changes in the total number of clusters especially in the middle of summer and generally it trends toward decreasing. Differently from potatoes in apples, the total number of clusters is less strongly depending on growth time.

For a better understanding, we analyzed some water clusters in detail where we started with $\left(\mathrm{H}_{2} \mathrm{O}\right)_{12}$. In Figure 5, the degree of individuality or how this cluster interacts with its surroundings $(f)$ in dependence on season time is given. In both potato cultivars, the $\left(\mathrm{H}_{2} \mathrm{O}\right)_{12}$ dynamics are nearly synchronous during growth. As visible, on 01.07.2006 the cluster lost almost completely its individuality to explain by a strong interaction with the bio-matrix of the potato tissue. After that the interaction weakens and the cluster individuality increases up to $12 \%$. Beginning with 01.08.2006 there aren't any important changes. In apples, the cluster $\left(\mathrm{H}_{2} \mathrm{O}\right)_{12}$ possesses a much higher individuality than in potatoes that is visible as a maximum at 12.08 .2006 (up to $15 \%$ ). In potatoes this cluster interacts more strongly with surroundings in the bio-matrix than that one in apples. Possible cluster interaction with surroundings is shown in Scheme 2.

The interaction of the cluster consisting of 100 water molecules with its surroundings is given in Figure 6. This water cluster is in the collapsed form only during the indicated growth period. The interaction with surroundings is very weak $(|f|>>0)$. The curves for both two potato cultivars have two peak areas where the cluster is active at the interaction with surroundings. The peak maxima are on July $15^{\text {th }}$ and August $10^{\text {th }}$ (arrows). Both peaks can be compared with those in Figure 5, but they are shifted for one month to a later time. For the $\left(\mathrm{H}_{2} \mathrm{O}\right)_{100}$ cluster in apples, there was found a broad peak between July $15^{\text {th }}$ and August $10^{\text {th }}$. In this time range, the individuality reaches nearly zero indicating an unknown influence on the cluster formation on the part of the surroundings in the bio-matrix (see Scheme 2). Thus, similar processes involving cluster $\left(\mathrm{H}_{2} \mathrm{O}\right)_{100}$ proceed both in potatoes and in apples.

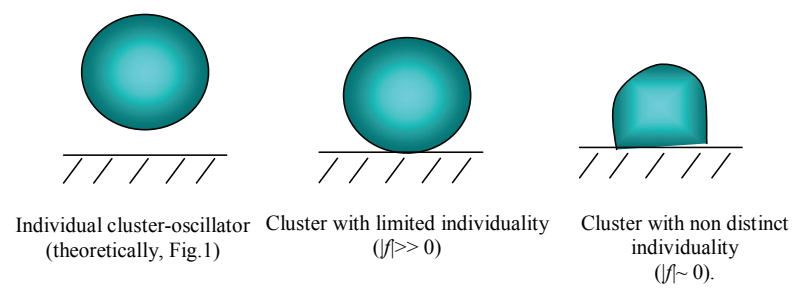

Scheme 2 


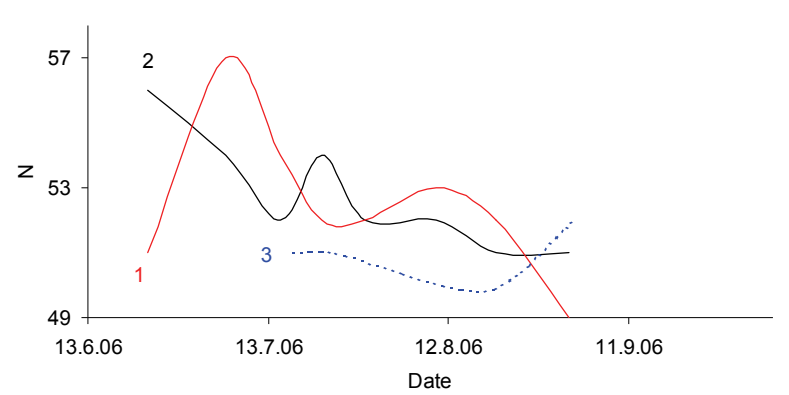

Figure 4. Number of cluster kinds in: 1 - tubers of Agria, 2 tubers of Kalena, 3 - apple.

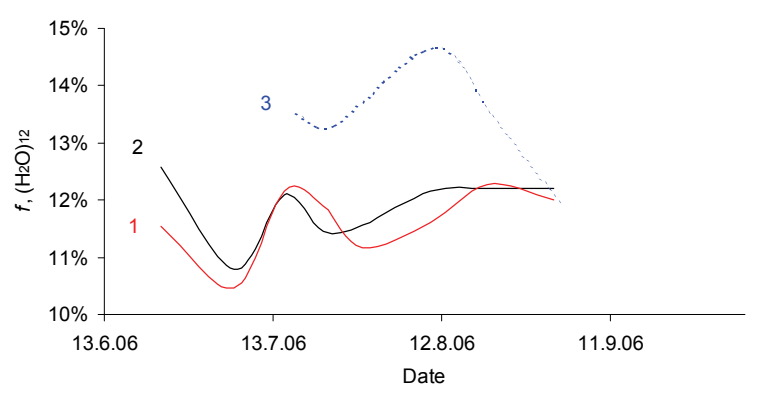

Figure 5. Relative rate of the water cluster $\left(\mathrm{H}_{2} \mathrm{O}\right)_{12}$ in: $1-$ tubers of Agria, 2 - tubers of Kalena, 3 - apple.

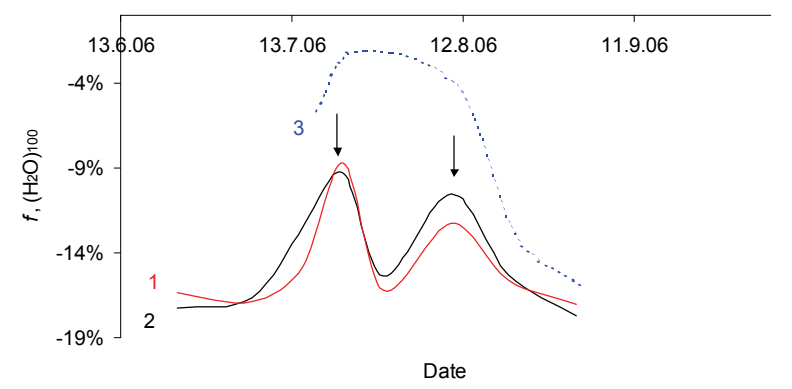

Figure 6. Relative rate of the water cluster $\left(\mathrm{H}_{2} \mathrm{O}\right)_{100}$ in: $1-$ tubers of Agria, 2 - tubers of Kalena, 3 - apple.

In the following these investigations were applied to the water cluster $\left(\mathrm{H}_{2} \mathrm{O}\right)_{178}$ (Figure 7), that exists in the expanded form only. The curves are similarly with two maxima on July $15^{\text {th }}$ and August $20^{\text {th }}$. Compared with the two potatoes cultivars the curve for apples shows higher $f$-values due to a stronger individuality of the clusters that means these clusters' interactions with the surroundings is lower. On the other side the behavior of all three curves is similarly and reflects a general biochemical processes in plants (marked by arrows), possibly. However, computer animation did not give this cluster, by Chaplin [10] the clusters consisting of 160 and 184 water molecules were computed only. To the authors the cluster $\left(\mathrm{H}_{2} \mathrm{O}\right)_{178}$ is caused by bio-matrix influence. The mass analog of this cluster was found for polysaccharides extracted from plants during the time from July $15^{\text {th }}$ to August $20^{\text {th }}$ too.

The next discussion concerns the water cluster of 280 molecules (Figure 8), that is present in the collapsed form only in all investigated plants. Independently on plant type for this cluster, we recognized clear individuality between July $15^{\text {th }}$ and August $20^{\text {th }}$. Outside this time interval that means until July $15^{\text {th }}$ and after August $15^{\text {th }}$ all curves approaches to the zero line (x-axe). The cluster seems to react on changes in the bio matrices too. The influence of bio matrix leads to a dominating collapsed form. Later the August $15^{\text {th }}$ ageing processes in potatoes and apples cause bio matrix compression. The biopolymers contract and press out included water molecules, comparably with the gel-xerogel transformation. The $\left(\mathrm{H}_{2} \mathrm{O}\right)_{280}$ clusters shall be destroyed by densification of the bio matrix in ripe plants.

Figure 9 is shown how the formation energy of this cluster changes during the plant growth. $\mathrm{E}_{\text {form }}$ varies in the interval between 0.4 and $1.3 \mathrm{~kJ} / \mathrm{mol}$ and achieves its highest value in the end of July. This result agrees satisfactorily with the formation energy for the cluster in fresh distilled water $(0.4 \mathrm{~kJ} / \mathrm{mol})$ [1] and with the experimentally observed value of $\sim 1 \mathrm{~kJ} / \mathrm{mol}[10,13]$.

Finally, we shall study the water cluster $\left(\mathrm{H}_{2} \mathrm{O}\right)_{1889}$, that was calculated by Chaplin [10] too (Figure 10). There are strong differences between the oscillations in the two potato cultivars on the one hand and in the apples on the other hand but generally, the $f$ value changes were found

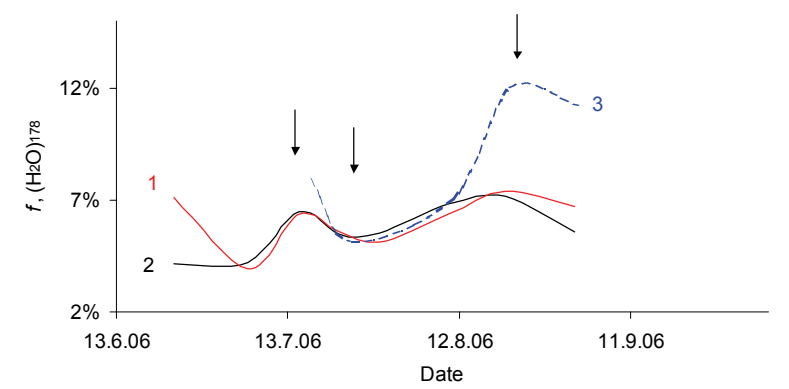

Figure 7. Relative rate of the water cluster $\left(\mathrm{H}_{2} \mathrm{O}\right)_{178}$ in: 1 tubers of Agria, 2 - tubers of Kalena, 3 - apple.

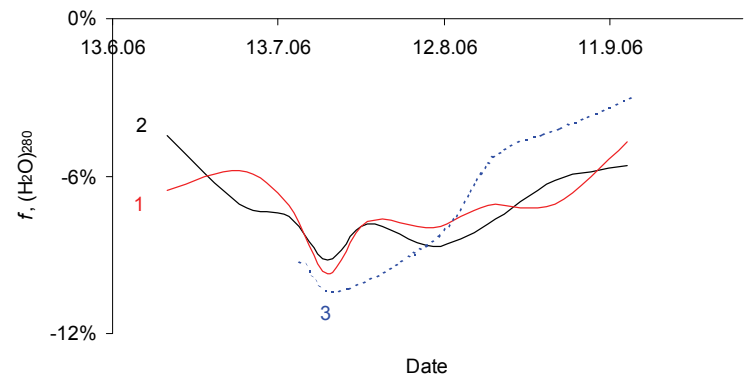

Figure 8. Relative rate of the water cluster $\left(\mathrm{H}_{2} \mathrm{O}\right)_{280}$ in: 1 tubers of Agria, 2 - tubers of Kalena, 3 - apple. 


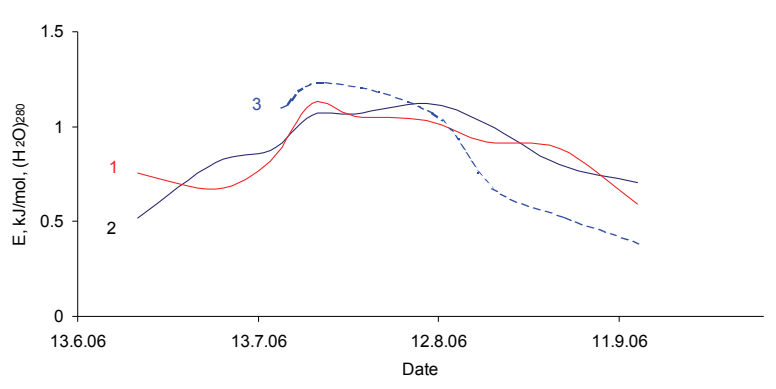

Figure 9. Changing formation energy of $\left(\mathrm{H}_{2} \mathrm{O}\right)_{280}$ in plants during growth. 1 - tubers of Agria, 2 - tubers of Kalena, 3 - apple.

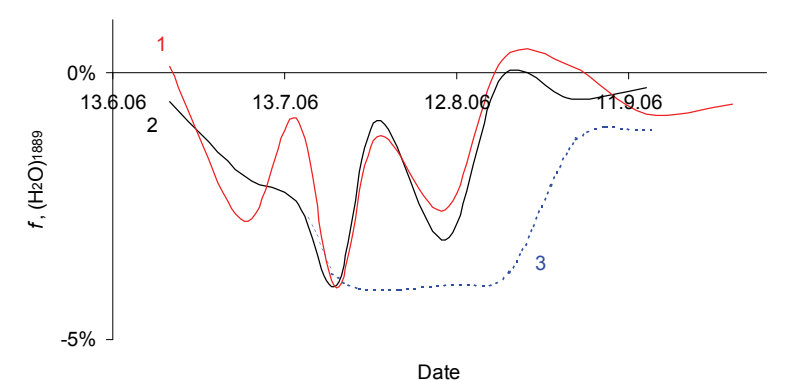

Figure 10. Relative rate of the water cluster $\left(\mathrm{H}_{2} \mathrm{O}\right)_{1889}$ in: $1-$ tubers of Agria, 2 - tubers of Kalena, 3 - apple.

to be lower than for the other smaller clusters $(-4 \%)$. In potatoes, the interaction of this cluster with the surroundings during growth seems to be very sensitive and in September the signal disappeared completely, which applies for apples too.

The average molecular mass of all clusters in plants during growth is represented in Figure 11. By comparing it with Figures $\mathbf{6}$ and $\mathbf{8}$ it can be concluded that the behavior of the average molecular mass of clusters in dependence on the time shows some similarities with signals of $\left(\mathrm{H}_{2} \mathrm{O}\right)_{100}$ and $\left(\mathrm{H}_{2} \mathrm{O}\right)_{280}$. Remarkably, that the absolute maxima for all curves are at the same time. It could be an indication for that similar biochemical processes on the level of super molecular structures proceed in the bio matrix of all investigated plants. Comparing the results of Figures 6, 7 and $\mathbf{1 1}$ at least 2 to 3 transformation processes in plants during growth can be concluded.

Based on the results discussed above the following model for water cluster forms in bio matrices of potato tubers has been suggested. In this model (Figure 12), the bio matrix is represented as line net and water clusters as balls. It is visible how the changing bio matrix influences the water cluster form. In young potato, $\left(\mathrm{H}_{2} \mathrm{O}\right)_{100}$ and $\left(\mathrm{H}_{2} \mathrm{O}\right)_{178}$ are in the collapsed form whereas $\left(\mathrm{H}_{2} \mathrm{O}\right)_{280}$ and $\left(\mathrm{H}_{2} \mathrm{O}\right)_{1889}$ are in the expanded one (dotted line). In the middle of summer the bio matrix growths very fast; which leads to large pores and the pressure on the part of the bio matrix on the water clusters becomes weaker. In

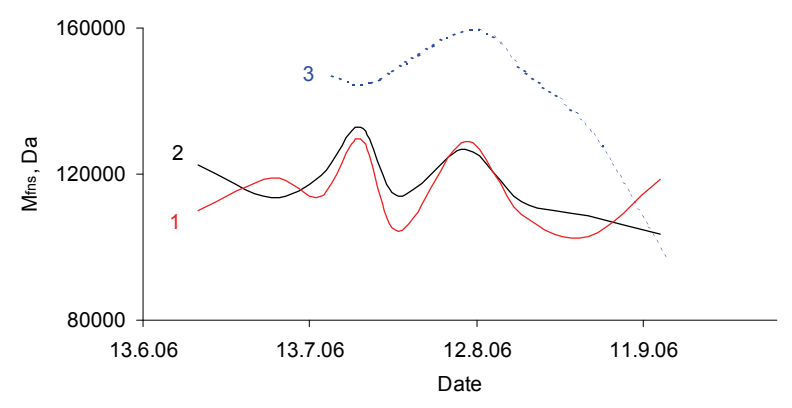

Figure 11. $M_{\text {fns }}$ of all clusters in vivo versus the growth time: 1 - tubers of Agria, 2 - tubers of Kalena, 3 - apple.
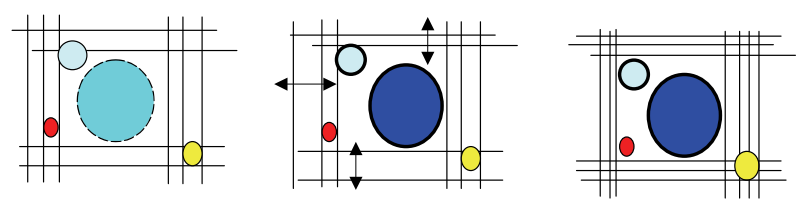

$$
\begin{aligned}
& \text { water clusters in } \\
& \text { bio matrix of } \\
& \text { young potato }
\end{aligned}
$$

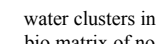

bio matrix of no

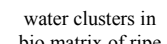

bio matrix of ripe potato
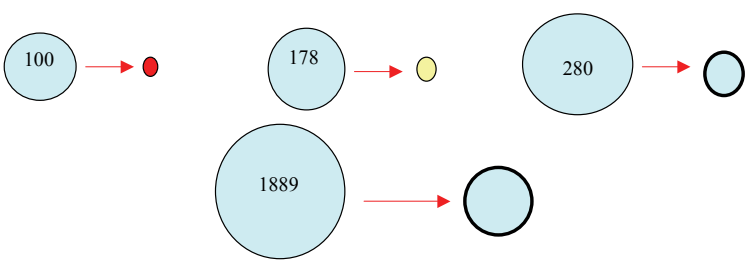

Figure 12. Model of water cluster interaction with bio matrix in dependence on growth time.

young plants, the individuality of clusters is more developed. With plant ripening, the bio matrix loses water due to, that all super molecular structures contract and release water in the plant tissue like gel xerogel transformation (syneresis).

For a better understanding of the bio matrix influence on the water clusters, amylopectin was extracted during the potato growth and the $M_{\text {fns }}$ values were measured and compared with the $\mathrm{M}_{\text {fns }}$ values of all clusters in the potato tissue (Figure 13). The $\mathrm{M}_{\text {fns }}$ of amylopectin (grape-like clustering) is much higher than the corresponding value for all clusters in the potato tissue. Curve 1 as well as curve 2 has two maxima, though concerning their position there are some differences. The first maximum (marked with a star) in the curve 1 on July $6^{\text {th }}$ corresponds with the minimum in the curve 2 on the other side, the second maximum of the curve 1 (August $10^{\text {th }}$ ) clearly correlates with the second maximum in the curve 2 . The minimum (July $22^{\text {nd }}$ ) in curve 1 shall be explained with a debranching process of amylopectin at temperatures higher $298 \mathrm{~K}$ [14]. The similarities between both curves assume that the two events, which takes place in amylopectin (bio matrix) and water clusters in the potato tissue are interconnected. 


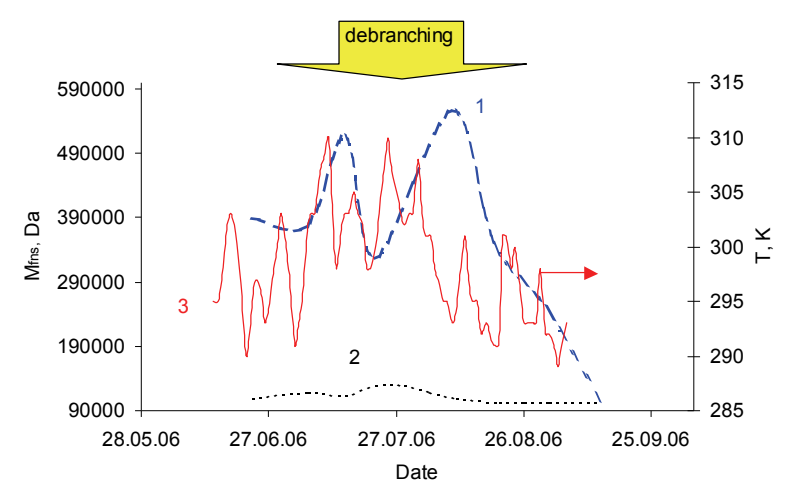

Figure 13. Average molecular masses $\left(\mathrm{M}_{\mathrm{fns}}\right)$ of amylopectin super coils isolated from the potatoe cultivar Agria (1) and of all clusters in potatoe tuber tissue of Agria in vivo (2), the average day temperature line (3).

In the next, briefly some small water clusters shall be compared with $\mathrm{M}_{\text {fns }}$ of amylopectin (amorphous area) as a possible representative of the macromolecular structure in the bio matrix (Figure 13).

Both in young and ripe potatoes, the water cluster $\left(\mathrm{H}_{2} \mathrm{O}\right)_{100}$ is in a free undisturbed state (Figure 6) whereas during the growth (not ripe state, Figure 12) when the bio matrix changes a new interaction between this cluster and the bio matrix leads to a new cluster form. Both sides seem to influence each other (see the correlation between curves 1 and 2 in Figure 6 and curve 1 in Figure 13). In contrary, $\left(\mathrm{H}_{2} \mathrm{O}\right)_{178}$ doesn't participate in active biochemical processes in the bio matrix (bad correlation between the curves 1 and 2 in Figure 7 and curve 1 in Figure 13) due to that it is actively involved in the super molecular structure net of the bio matrix which retains this cluster.

Only on July $22^{\text {nd }}$ shows the collapsed cluster $\left(\mathrm{H}_{2} \mathrm{O}\right)_{280}$ a strong individual behaviour in potatoes and apples (Figure 8), but in young and old plants the bio matrix destroys the cluster, the $f$ - value approaches to zero (Figure 12).

Concerning the bio matrix influence on large clusters, the $\left(\mathrm{H}_{2} \mathrm{O}\right)_{1889}$ is the most sensitive one (Figure 8). During the growth it is permanently forced to change its form (Figure 12).

For the integral distribution of clusters in plants we found the following equation:

$$
\mathrm{W}_{\mathrm{x}}=a \cdot \ln \left(m \cdot 10^{-5}\right)+b,
$$

where $\mathrm{W}_{\mathrm{x}}$ - the integral rate of cluster fractions, $m-$ the mass of clusters in Dalton, the value of coefficients $a$ and $b$ change depending on the ripe degree. We describe these changes with the following equations for apple ("elstar", $83 \pm 2$ wt. \% water in ripe state):

$a=-5 \mathrm{E}-09 \cdot t^{4}+5 \mathrm{E}-05 \cdot t^{3}-0.1816 \cdot t^{2}+295.49 \cdot t-180235$,

$b=6 \mathrm{E}-09 \cdot t^{4}-6 \mathrm{E}-05 \cdot t^{3}+0.2311 \cdot t^{2}-378.76 \cdot t+232739$,

where, $t-$ date of the test.

For potato tubers, the character of $a$ and $b$ changes cannot be described with these equations. The values $a$ and $b$ strongly depend on the ripe time and they reflect some changing processes in super molecular structures in tuber tissue during potato growth.

The signal frequencies $(\mathrm{kHz})$ we detected directly for the water clusters in tubers of two potato cultivars and one apple cultivar consisting of $11 \pm 1,100,178,280,402,545$, $715,903,1119,1351,1606$ and 1889 molecules were: 22.2 , 7.3, 5.5, 4.4, 3.7, 3.1, 2.8, 2.4, 2.2, 2.0, 1.8 and 1.7.

\subsection{Water Cluster Communication Field. A Channel of Plant Communications}

Now we shall discuss a hypothetical oscillation model of one element being part of a multi element system (cluster like dimension). The oscillation of one molecule (P) can be envisaged as a pendulum and the oscillation of the dimension of $\mathrm{P}$ like a dimension of pendulums (PD), oscillating in one rhythm (minimization of the total energy and harmonisation of the model, Scheme 3 ).

In these systems, the pendulum oscillations depend both on the nature of the pendulum and its interaction with surroundings. The driving force (DF) of the association of some $\mathrm{P}$ in $\mathrm{PD}$ is the resonance that harmonizes the whole system leading to a higher stability and to a minimum of the total energy in the new ensemble. Furthermore, PD oscillations influence other oscillation systems in their surroundings by resonance, where stronger PD forces weak PD to oscillate in the rhythm of the strong PD. How strongly the surroundings shall be influenced by PD depends on the number of $\mathrm{P}$ in the ensemble. The information transfers from PD to P and backwards proceeds through the medium in which are pendulums.

It would be interesting to investigate the information transfer between clusters of water (PD) being in different vessels and of water in a model biological tissue (hydrogel). Agarose hydrogels are often used in biochemistry and microbiology where their chemical structure is well known and they can be considered as biological tissue $[15,16]$. If this information transfer channel exists really then it should be observed by analysing the influence of one $\mathrm{PD}_{\mathrm{A}}$ (vessel $\mathrm{A}$ ) on the other one $\left(\mathrm{PD}_{\mathrm{B}}\right.$, vessel $B)$. For that the water cluster ensembles in vessel $A$ were stimulated by a forced stirring of water (destruction of long-range order) contemporaneously the water in vessel $\mathrm{B}$ at the distance $x$ was in a calm state. The interaction of cluster ensembles belonging to water in different vessels was measured with help of the Zubow gravitation spec-

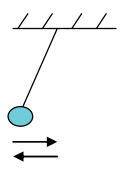

(P)

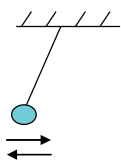

Scheme 3
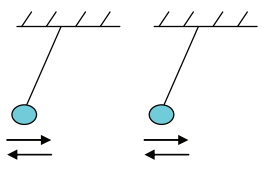

(PD) 
trometer given in Figures 14 and 15 [6].

Water clusters like oscillators or pendulum ensembles have to communicate with each other by a resonance field. Here, one dimension of a water cluster-pendulum in vessel A (Figure 14) interacts with an other one of the same water cluster-pendulum in vessel B by the resonance between these pendulums (Scheme 2) where the resonance field is not homogenous, since it is influenced by a number of other surrounding resonators (water clusters of the neighboring river, water in floor, wood, clouds etc.). To check it, a simple experiment was carried out.

Before beginning the experiment and to achieve a thermal and mechanical balance the system was stored for $6 \mathrm{~h}$ excluding the influence of outer thermal flow, light and mechanical fields. At this time the characteristics of long-range order in water (Figure 14), agarose hydrogel and potato tissue (Figure 15) in which the GS-sensor was installed were measured three times. Then, water in vessel B was stirred for 1 min by a Teflon stirrer, the electrical engine was switched off and after 1 min the GS-spectra were taken in vessel A in an interval of $5 \mathrm{~min}$. As visible in Figure 14, forced destruction of the long-range order in water in vessel $\mathrm{B}$ changes the long-range order in water of vessel A (strong increase of $\left.\mathrm{M}_{\mathrm{fns}}\right)$. This reaction supports the correctness of Scheme 2. According to Scheme 2 water clusters as oscillator ensembles interact each with other by resonance. We found that water clusters differently react on forced activation of their analogous in vessel B. Shortly after at $30 \mathrm{sec}$ a maximum $\mathrm{M}_{\text {fns }}(250 \mathrm{kDa})$ was achieved, $\mathrm{M}_{\text {fns }}$ strongly decreased to $120 \mathrm{kDa}$ and approximated then to a stable level.

The rare cluster $\left(\mathrm{H}_{2} \mathrm{O}\right)_{137}$, is the most sensitive one to the resonance field influence of water that is placed in an other vessel, it appears and disappears. The vibration process lasts 5 minutes and is comparable with a decreasing oscillation.

Hence by the integral characteristics of the long-range order in liquids, e.g. $\mathrm{M}_{\text {fns }}$, changes in the fine cluster structure cannot be analyzed for the observer the process was finished after 1 minute. At the level of individual clusters, to get an answer on the excitation from analogous in the other vessel can pass some minutes or even hours.

The question raised is whether water clusters, for instance in agarose hydrogel or in plant tissue (receiver), can communicate with cluster ensembles in stirred water (transmitter) that is at a distance of $18 \mathrm{~cm}$ and $31 \mathrm{~cm}$. To give an answer on this question the next equipment was developed (Figure 15).

These communication signals (change of the average mass of the secondary activated water clusters in the receiver cell) are shown in Figure 16. In the first 10 minutes when the stirrer is switched off $\mathrm{M}_{\mathrm{fns}}$ is nearly

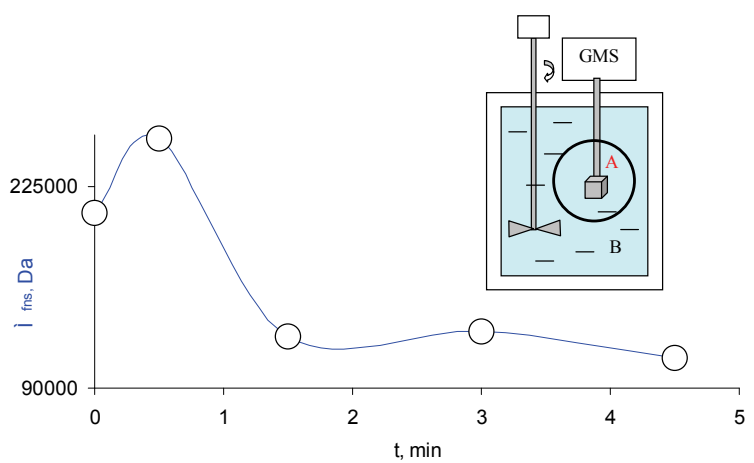

Figure 14. Change of $\mathrm{M}_{\text {fns }}$ in vessel A by stirring water in vessel B. Both vessels are filled with distilled water, $286 \mathrm{~K}$. The stirring engine at GS-measuring was switched off. The equipment was isolated from light and mechanical vibrations.

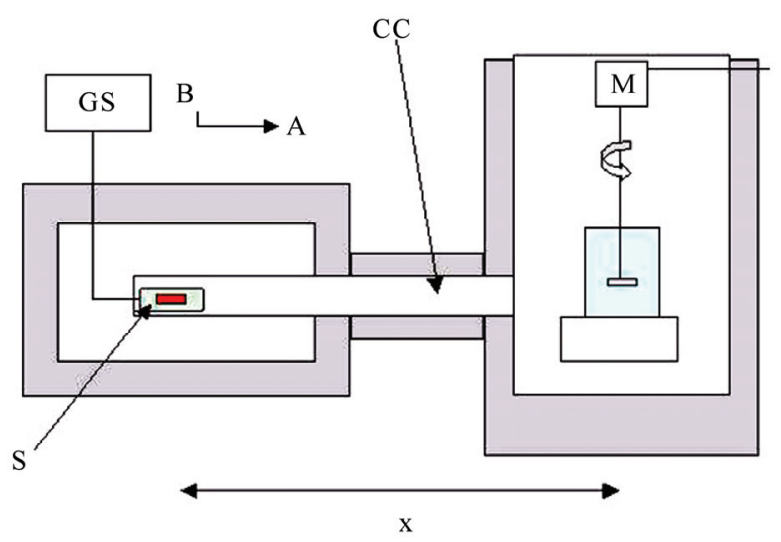

Figure 15. Scheme for the determination of the information field caused by the interaction of water clusters that are distant of each other. $286 \mathrm{~K}, \mathrm{x}=18$ and $31 \mathrm{~cm}$. GS-sensor and water-stirring equipment were placed in a sound isolated polystyrene foam containing box. Before each GS measurement (1 min) the stirring engine (M) was switched off. A - direct vessel communication by the communication channel ( $\mathrm{CC}$, pipe), $\mathrm{B}-$ contact-free variant ( $\mathrm{CC}$ is directed to the line of the shortest interaction with an angle of $90^{\circ}$ ) $\mathrm{S}$ - sample (GS-sensor is in 3 wt. \% agarose gel or directly in tuber tissue).

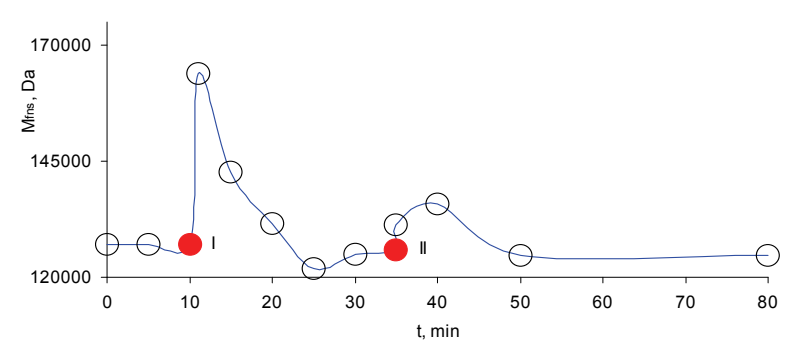

Figure 16. Effect of directed resonance interaction of water clusters in the measuring box with water clusters in the stirring vessel (Figure 14). I - measuring after 1 minute stirring and 1 minute break, directed position (Figure 14 in the direction of A), II - measuring after 1 minute stirring and 1 minute break, non-directed position (Figure 14 in the direction of B). 
unchanged then, one minute stirring causes (transmitter) a strong increase of $\mathrm{M}_{\text {fns }}$ in the agarose hydrogel (receiver cell). This signal is unsymmetrical, while the increase is very steep the decrease proceeds more slowly. Based on this a wireless communication between these two systems, between transmitter (activated water) and receiver (water clusters in agarose hydrogel) was concluded. The same stirring procedure was repeated 10 minutes later and as visible the answer is similarly but of a lower intensity.

Analogous experiments were carried out with potato tubers in which the GS-sensor was installed (Figure 15). As for agarose hydrogel for the potato bio matrix (in-vivo) an answer reaction on the forced destruction of long-range order in water in a vessel that is at a distance of $18 \mathrm{~cm}$ was observed too (Figure 17). The test was made three times. The first peak is symmetrical though comparing with agarose hydrogel the decrease of $\mathrm{M}_{\mathrm{fns}}$ is deeper. The second and third effects are similarly. The triple repeated experiments support clearly that there exists a communication channel between water clusters in tubers and water clusters of the surroundings.

Several water clusters in potato tubers differently react on impact of resonance field caused by the transmitter (forced stirred water in vessel), some clusters react fast within some seconds whereas other need more time (range of some minutes).

The strong increase of $\mathrm{M}_{\text {fns }}$ and then its fast falling down lower the $\mathrm{M}_{\text {fns }}$ level in a tuber without field influence indicate that this influence can be regarded as a shock wave, that consists of a falling and a reflective wave and that is comparable with low tide and high tide. The energy flow from activated clusters (see Figure 18) achieves the water clusters in the potato tuber after that the average cluster mass in the bio matrix increased. This process is characterized by condensation of small clusters and domination of large one.

In the following 15-20 min, after the energy impact and energy dissipation in the bio matrix thermodynamic balance has been recovered (Figure 17). The elasticity of this interaction can serve as characteristic for the stability of the bio matrix against the influence of fields caused by other molecular oscillators in the hydrosphere of the earth (natural and artificial irrigation, influence of large water masses, e.g. clouds).

The detail study of resonance processes of some clusters has shown that new clusters appear whereas old ones disappear finally, leading to changed surroundings by different clusters. The rare water cluster containing 226 molecules is formed at forced stirring of water [17]. In the potato tuber it is present too though the signal is of low intensity $(f=0.1 \%)$ and it appears only after the third impact at 2750 seconds (Figure 17). Its oscillation intensity first increases eight times, then after $125 \mathrm{sec}-$ onds (2875 s, Figure 17) it decreases 38-times, later

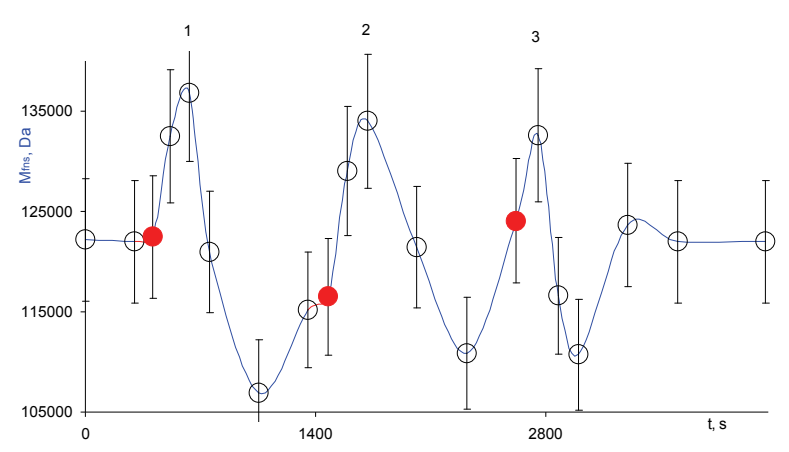

Figure 17. Change of the average molecular cluster mass in a potato tuber (in-vivo) under the resonance field influence of oscillating water clusters in a $2 \mathrm{~L}$ water vessel that is at a distance of $18 \mathrm{~cm}$ (Figure 15), $286 \mathrm{~K}$. Communication medium air. The black circle means the switching on of the stirrer for 1 minute. The spectra were taken when the engine was turned off.

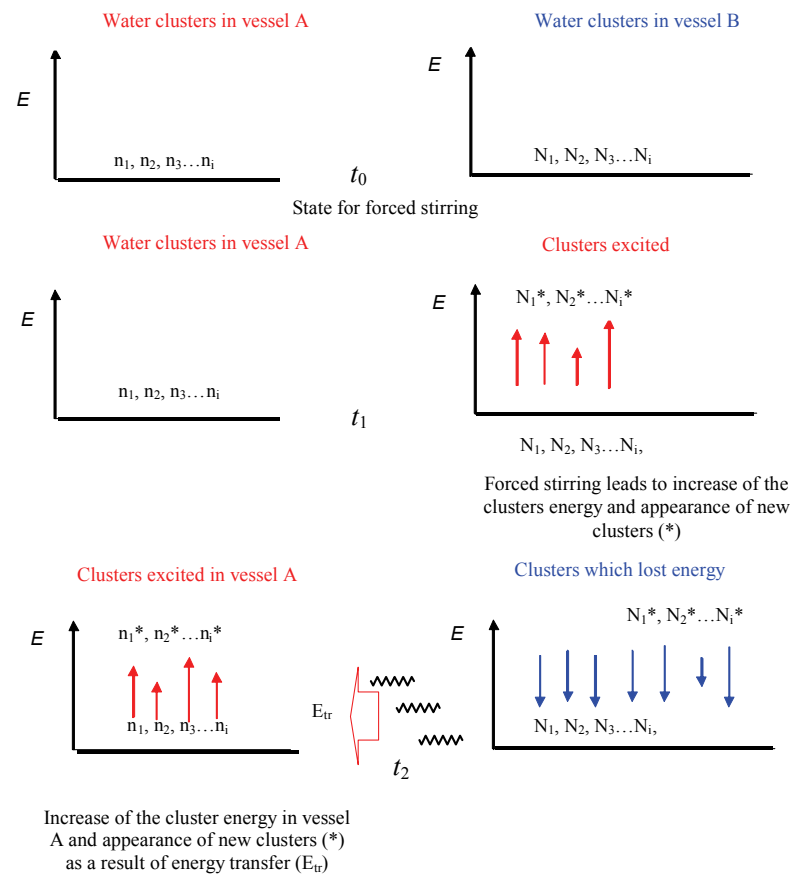

Figure 18. Scheme illustrating how the energy levels of water clusters in hydrogel were filled by energy transfer. Each oscillator is marked by $\mathrm{n}_{1}, \mathrm{n}_{2}, \mathrm{n}_{3} \ldots \mathrm{n}_{\mathrm{i}}$ (number of water cluster kinds in hydrogel) and by $\mathrm{N}_{1}, \mathrm{~N}_{2}, \mathrm{~N}_{3} \ldots \mathrm{N}_{\mathrm{i}}$ (number of water cluster kinds in forced stirred water). The energy levels of water clusters in hydrogel will be filled as soon as the energy $\left(E_{t r}\right)$ is delivered from stirred water.

after $245 \mathrm{~s}(2995 \mathrm{~s})$ it increases again 27-times and after $1385 \mathrm{~s}$ (4136 s) it calms down while the other clusters react five times weaker. Remarkably, this cluster is present in the communication net agarose hydrogel - water too (Figure 15), but it isn't in the water-water system (Figure 14). In the water-water system the most active role played an other cluster, namely $\left(\mathrm{H}_{2} \mathrm{O}\right)_{137}$. The acti- 
vation of $\left(\mathrm{H}_{2} \mathrm{O}\right)_{226}$ seems to be caused by the presence of polysaccharides in the bio matrix. We assume that this cluster is integrated in the bio matrix pores of the tuber tissue and that it leaves these pores under the impact of shock waves where this process is reversible (Scheme 4).

The appearing of the new unstable state in the tuber bio matrix (Scheme 4, right part of equation) gives a signal to the plant, which can be interpreted as the beginning of the irrigation, the approaching of rain clouds or the rain beginning. As the consequence the plant switches on a preparation mechanism for irrigation. Analogous communication between plants and their surroundings at approaching of drought, frost and winds etc., is possible under participation of other cluster signals.

Additional studies showed there is a fast communication channel in the system tuber-tuber, namely a mechanically destroyed tuber influences its neighbouring tuber in which is a GS-sensor installed (Figure 15).

The information field of water clusters' interaction in tubers is sensitive to the water quality namely rain, river or distilled water (Figure 3) as well as to neighbours containing a large amount of water and possessing a cluster field like a dimension of resonators (Scheme 3). This information channel diversity permits to use it for plant communication. The amount of communication channels can be estimated for the first nine members of the cluster sequence by the next equation:

$$
\mathrm{C}=\sum_{i=1}^{9}\left(\begin{array}{l}
9 \\
i
\end{array}\right)
$$

The equation gives 509 communication channels which can be multiplied by the number of kinds of super molecular structures $(n)$ for instance water clusters surrounding uniform bio molecules in the bio matrix which appears like a unique oscillator block. The whole information field capacity is the following:

$$
\mathrm{C}_{\mathrm{o}}=\mathrm{C} \cdot n \text {. }
$$

The transformation velocity is in the sound frequency area and depends on the traffic kind (cluster transmitter and cluster receiver) too, where the transformation quality can be influenced by external factors e.g. resonance fields of water clusters belonging to other systems.

For all clusters the oscillation frequencies are known therefore, it is possible to develop a program and equipment for an early warning system for plants about weather changes, parasite attacks additionally, for a system monitoring and stimulating the ripening process (Scheme 5).
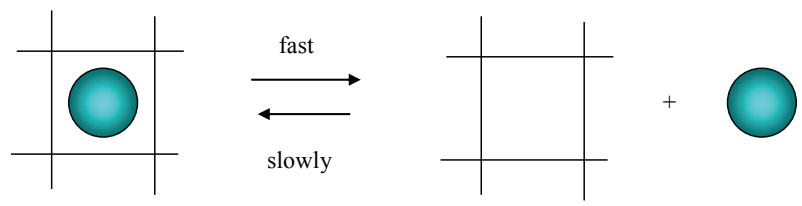

Scheme 4

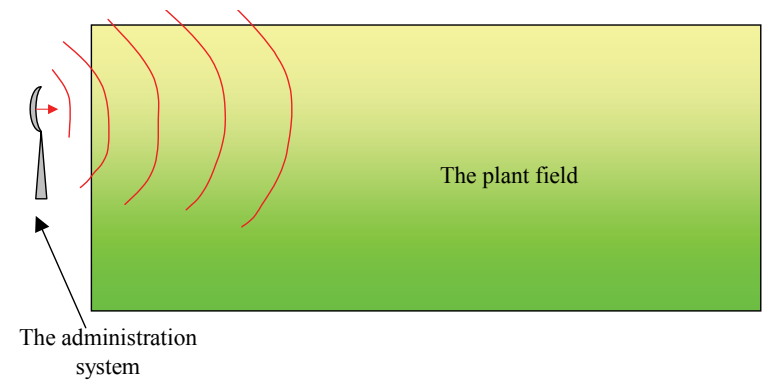

Scheme 5

In Figures 18 and 19, we shall represent the mechanism of a possible communication between biological systems realized by resonance fields of water clusters. We see two dimensions of equal oscillators (state $t_{0}$, Figure 18), that are divided of each other by a molecular space, for instance air. The forced activation of oscillators of one dimension (in vessel B, Figure 18) by e.g. pumping with potential energy causes a new energy set of clusters $\left(\right.$ state $\left.t_{1}\right)$ which release this energy $\left(E_{t r}\right)$ after the forced energy pumping was finished and the system achieved the starting thermodynamic state $\left(t_{2}\right)$. The released energy will be transferred as a gravitation energy wave to other dimension of oscillators where it causes an answer in the form of oscillation of the whole system as well as of some individual clusters of the same type. The Figure 18 can be applied for cluster communication in liquids as well as in bio matrices.

The influence of water clusters on the conformation of a bio molecule surrounded by different water clusters is shown in Figure 19 furthermore, it is shown how water clusters change the communication of the first bio molecule with a second one that is distant and that can belong to an other plant.

It was shown by [18] that water around a bio molecule change its conformation. Additionally, we found that at resonance interaction of water cluster ensembles with other analogous water cluster ensembles the weak ensembles adapt to the dominant ones. This adaptation process or harmonisations of water cluster oscillations force bio molecules in the receiver system to accept the conformation of dominating transmitter system. The consequence is that the receiver bio molecules have to participate in the same biochemical processes like the transmitter bio molecules. These processes change the biological and physiological behaviour of plants.

Practically, we observed this effect in summer 2006 for potatoes produced by "Cloke" GmbH in North Germany. The influence of the water quality on the green plant part was visible for the potato cultivar Agria the irrigation with underground water led to colour loss followed by immunity weakening. On places of the same potato field where the irrigation machine didn't work the green plant part had a dark green colour. According to Figure 3 the cluster structure of water used for the irrigation isn't suitable to the water cluster structure in the 


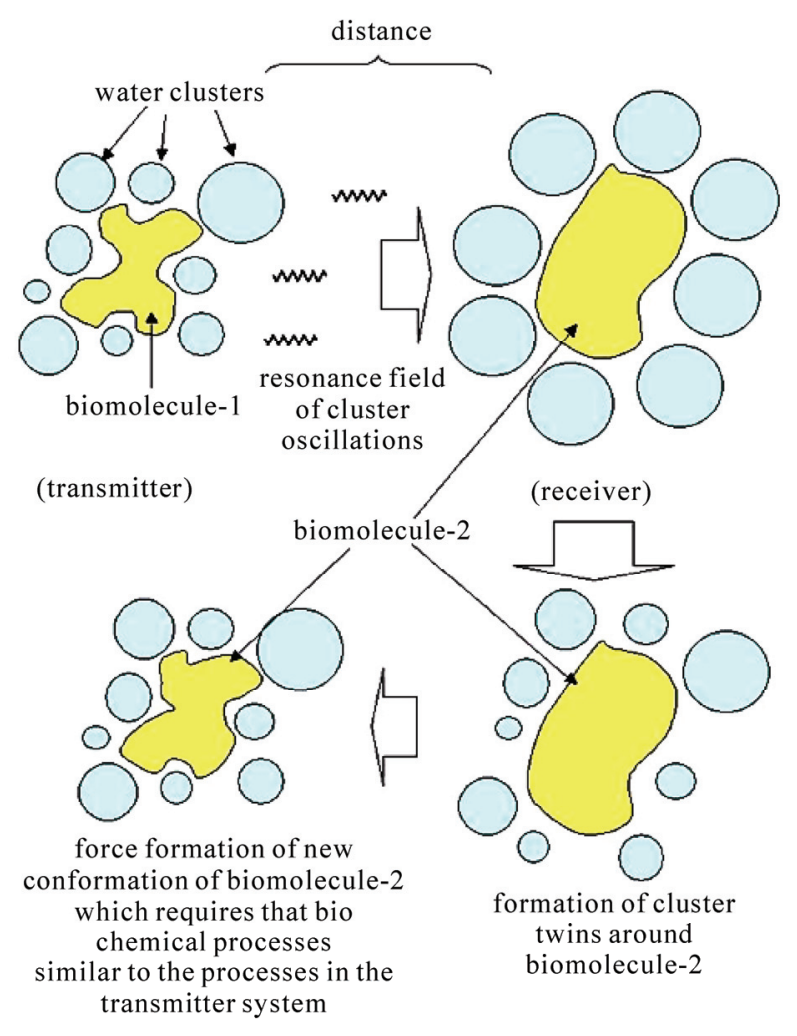

Figure 19. Model for information transfer from one bio molecule to an other one by forced change of the bio molecule conformation under the influence of water clusters.

plants. $\left(\mathrm{H}_{2} \mathrm{O}\right)_{12}(22.2 \mathrm{kHz}),\left(\mathrm{H}_{2} \mathrm{O}\right)_{178}(5.5 \mathrm{kHz}),\left(\mathrm{H}_{2} \mathrm{O}\right)_{280}$ $(4.4 \mathrm{kHz})$ are similarly for the potato tissue and rainwater. In contrary, $\left(\mathrm{H}_{2} \mathrm{O}\right)_{100}(7.3 \mathrm{kHz}),\left(\mathrm{H}_{2} \mathrm{O}\right)_{178}$ and $\left(\mathrm{H}_{2} \mathrm{O}\right)_{280}$ in the river and underground water are differently and oscillate in a opposite form to water in potato. This means that the plants need more energy for the adaptation of the irrigation water to the own water clusters.

Based on the oscillator interaction (Scheme 3) and the experimental proof for water, agarose hydrogel and potato tissue it was concluded that this effect could be applied for other plants.

Therefore, water clusters play an important role as a cost factor at the water choice. On the other side this damage can be reduced by a corresponding preparation of irrigation water, for instance, by influencing the artificial water cluster communication field or with help of physical measures or better by a correct choice of irrigation water.

It is shown in Figure 20 how the planets develop the relative molecular cluster energy in the interval $\boldsymbol{C}[6,12]$, in which masses and sizes of clusters are comparable with those of starch globular. In this once-only time, nearly all planets were together on one side to the sun. The surface of the 3D graphic is characterized by some regular maxima and minima, that correlate with planet influences (from left to right marked wit red arrows):

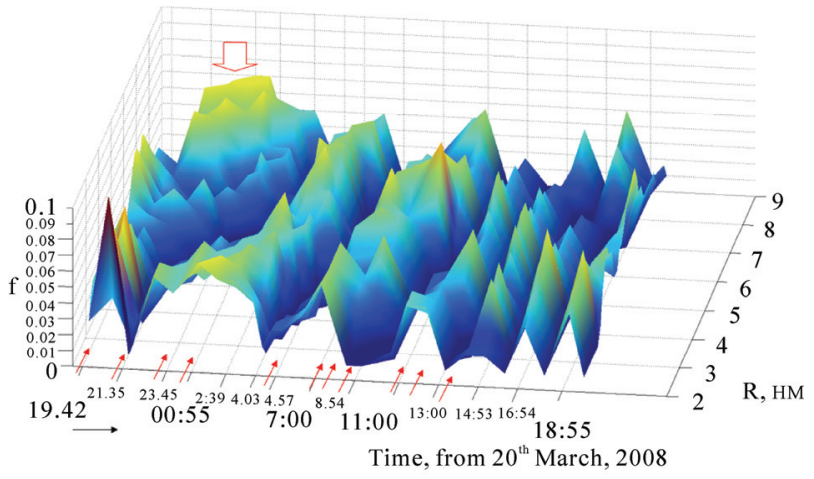

Figure 20. 3D graphic of GS spectra of potato bio matrix (interval $\mathrm{C}$ - for clusters of middle sizes) during fullmoon from March $20^{\text {th }} 2008$ (Berlin, summer time), $f$ - energy rate of a cluster in a cluster ensemle.

sunset at 19:19, Moon - Uranus opposition $\left(180^{\circ}\right.$ at $21: 28: 25)$ and Mars - Pluto opposition $\left(175^{\circ}\right)$ in the slice plane of proton resonance $[4,6]$. All these events led to strong changes in the energy characteristics of all cluster ensembles in the potato bio matrix (marked with a broad arrow). Further, the other arrows shall be designated to the appearance in this slice plane of: Saturn at 23:38, Moon at 00:39, second gravitation focal point of Moon orbital at 04:53, center of our Galaxy at 07:00, sunrice at $07: 17$, Pluto at 07:18, Jupiter at 08:35, Neptune at 10:56 and nearly simultaneously Mercury and Venus at 12:03, Uranus at 12:31 und Sun at 13:17.

Then, the signal intensities were highly decreased though with preserving the regularity. In $[4,6]$ we found out that planets influence the molecular matter by their gravitation radiation, that leads to a changed balance between proton dissolving in physical vacuum and proton condensation from physical vacuum. Thus, gravitation fields of planets were observed to impact strongly the cluster structure of molecules in bio matrices (longrange order) as well as consequently biochemical processes. These phenomena are signals for turning-on/off of biological cycles in the living matter.

\section{CONCLUSIONS}

Water in plants is present as clusters. These water clusters oscillate and communicate by oscillation with other water clusters of the surroundings. Water clusters and their distribution (collapsed or expanded) play an important role during plant growth. Their behavior in plants could be used as indicator for bio matrix development during plant growth. Plants communicate with each other and with surroundings by resonance field of oscillating water clusters. The right choice of irrigation water concerning the long-range order of water clusters could promote plant growth and reduce costs. Influence on plant communication by resonance field of water 
cluster oscillation could be applied for early warning systems on weather changes (drought, rain, frost, sun radiation etc.) or for regulation of biochemical processes during ripe, storage (inhibition of gel-xerogel transformation) as well as for manufacturing processes. Water clusters in plants were found to be unique sensors for the gravitation influence of planets.

\section{ACKNOWLEDGEMENTS}

The financial support by the, Aist Handels- und Consulting " $\mathrm{GmbH}$ (FNS-project) is gratefully acknowledged. The authors thank Dr. M. Reichmann (Institut für Pflanzenbau und Pflanzenzüchtung, Bayerische Landesanstalt für Landwirtschaft, FRG) for the kind consulting and supply of pure potato amylopectin samples.

\section{REFERENCES}

[1] Zubow, K.V., Zubow, A.V. and Zubow, V.A. (2005) Cluster structure of liquid alcohols, water and n-hexane. Journal of Applied Spectroscopy, 72(3), 321-328.

[2] Zubow, A.V., Zubow, K.V. and Zubow, V.A. (2007) Investigation of the distribution of water clusters in vegetables, fruits and natural waters by flicker noise spectroscopy. Biofizika, in Russian, 52(4), 585-592.

[3] Shoichi, O. (2004) Water structure in living organism. Mizu no Tokusei to Atarashii Riyo Gijutsu, 317-326.

[4] Zubow, K.V., Zubow, A.V. and Zubow, V.A. (2010) Principle of gravitation spectroscopy. New form of molecular matter. Processes. Fields. Electronic book available via Aist H\&C: http://www.zubow.de/

[5] Zubow, K.V., Zubow, A.V. and Zubow V.A. (2009) Low frequency movement of cluster-12 in potato amylopectin during growth. Influence of white noises. Journal Chemistry of Raw Plant Material, in Russian, 2, 81-88.

[6] Zubov, K.V., Zubov, A.V. and Zubov, V.A. (2010) Ensemble of clusters - new form of molecular matter, risks and chances. Zubow equations. In: Columbus, F., Ed., Chemical Industry Challenges - Issues and Prospects. Nova Science Publishers, Inc., Commack. http://www.novapublishers.com

[7] Bogdanov, E.V. and Mantrova, G.M. (2000) Equicluster water model. Biomedical Radioelectronics, in Russian, 7, 19-28.

[8] Lee, H.M., Suh, S.B. and Kim, K.S. (2001) Structures, energies and vebrational spectra of water undecamer and dodecamer: An ab initio study. Journal of Chemical
Physics, 114(24), 10749-10756.

[9] Lenz, A. and Ojamäe, L. (2006) On the stability of dence versus cage-shaped water clusters: Quantum-chemical investigations of zero-point energies, free energies, basis-set effects and IR spectra of $\left(\mathrm{H}_{2} \mathrm{O}\right)_{12}$ and $\left(\mathrm{H}_{2} \mathrm{O}\right)_{20}$. Chemical Physics Letters, 418(4-6), 361-367.

[10] Chaplin, M. (2009) Structural forms in the icosahedral water cluster. Available via South Bank University of London: http://www.lsbu.ac.uk/water/index.html

[11] Zubov, A.V., Zubov, K.V. and Zubov, V.A. (2007) Mechanism of sodium chloride diossolving in water and the process of the solution aging. Russian Journal of Applied Chemistry, 80(7), 1249-1255.

[12] Zubow, K.V., Zubow, A.V. and Zubow, V.A. (2008) Use of flicker noise spectroscopy for undestroyed analysis of nano structures. Diagnostics of Material, in Russian, 74(9), 40-46.

[13] Lee, H.M., Suh, S.B. and Kim, K.S. (2001) Erratum: Structures, energies and vibrational spectra of water undecamer and dodecamer: An ab initio study. [Journal of Chemical Physics, 114, 10749 (2001)]. Journal of Chemical Physics, 115, 7331-7334.

[14] Batlle, N., Carbonell, J.V. and Sendra, J.M. (2000) Determination of depolymerization kinetics of amylose, amylopectin, and soluble starch by aspergillus oryzae - amylase using a fluorimetric 2-p-toluidinylnaphthalene-6sulfonate/flow-injection analysis system. Biotechnology and Bioengineering, 70(5), 544-552.

[15] Kadri, A., Lorber, B., Charron, C., Robert, M.C., Capelle, B., Damak, M., Jenner, G. and Giege, R. (2005) Crystal quality and differential crystal-growth behaviour of three proteins crystallized in gel at high hydrostatic pressure. Acta Crystallographica, Section D: Biological Crystallography, D61(6), 784-788.

[16] Zhang, J.L., Takayama, H., Matsuba, T., Jiang, R. and Tanaka, Y. (2003) Induction of apoptosis in macrophage cell line, J774, by the cell-free supernatant from Pseudomonas aeruginosa. Microbiology and Immunology, 47(3), 199-206.

[17] Zubow, V.A., Zubow, K.V. and Zubow, A.V. (2006) The phenomenon of water clusters distribution in water and solvated clusters of ion pairs of $\mathrm{NaCl}$ in solution near the wall. The wall effect. Journal of Chemical Industry, in Russian, 83(6), 300-306.

[18] Shirayev, A., Pagan, D.L., Gunton, J.D., Rhen, D.S., Saxena, A. and Lookman, T. (2005) Role of solvent for globular proteins in solution. Journal of Chemical Physics, 122(23), 234911. 